\title{
Models of reading performance in older adults with normal age-related vision
}

\author{
Rolf W. Nygaard, PhD; ${ }^{1}$ Katharina V. Echt, PhD; ${ }^{1-2}$ Ronald A. Schuchard, PhD; ${ }^{1,3^{*}}$ \\ ${ }^{1}$ Atlanta Department of Veterans Affairs Rehabilitation Research and Development Center of Excellence, Atlanta, GA; \\ Departments of ${ }^{2}$ Medicine and ${ }^{3}$ Neurology, Emory University School of Medicine, Atlanta, GA
}

\begin{abstract}
Reading rate with print size (reading performance) was studied in a group of 132 older readers with normal agerelated vision using the Minnesota Low Vision Reading Acuity (MNREAD) test. Regression by a monotonic Weibull model had greater convergence success with lower residual error than either Logistic or Gompertz models. Reading performance by Weibull model regression was characterized by inflexion slope, critical print size, and maximum reading rate. Successive shortening of the data set length before regression by the monotonic Weibull model gave decreased fitting error. This finding suggests that some individual reading rates, rather than asymptoting at the largest print size, may give nonsigmoidal responses. Shortening the data set length decreases regression error but significantly changes regression parameter values. A nonmonotonic Weibull model that was sensitive to declining reading rate at large print size improved regression on $22 \%$ of our data. This result indicates that a subgroup of subjects had response falloff at large print size and reading performance characteristics that included incremental and decremental reading rate slopes at different print sizes, which were separated by a reading rate plateau.
\end{abstract}

Key words: Gompertz model, growth models, Logistic model, MNREAD, nonlinear regression, normal readers, reading, reading rate, rehabilitation, sigmoidal models, Weibull model.

\section{INTRODUCTION}

One measure of reading performance is reading rate of printed text of varying sizes [1-2]. Individual variation of visual nystagmus, eyeblinks, comprehension/literacy, and sundry features of entoptic and nonentoptic origin provide the noise background within which reading rate is embedded. The reader's task involves detection and discrimination of a signal, similar to that required in studies of auditory detection or visual discrimination, where data (appropriately scaled) often take sigmoidal form [34]. Therefore, assuming that reading rate versus print size has a sigmoidal relation is reasonable.

For each reader, we can also reasonably assume that at each print size a population of reading rate values exists that is normally distributed about the population means [5]. If data samples are distributed about those means, their regression (which converges on the mean) simulates reading rate across print size and gives a psychometric template. This template may be used to calculate reading rate characteristics like change in reading rate with respect to print size (slope), smallest print size at maximum reading rate (critical print size [CPS]), average maximum reading rate (plateau), the point where reading rate slope is greatest (inflexion), and the point where print size is so large that reading rate decreases [1-2].

Abbreviations: $\mathrm{CPS}=$ critical print size, $\mathrm{MNREAD}=$ Minnesota Low Vision Reading Acuity, SR = squared residuals, SSE = sum squared error, RMSNE = root-mean-square normalized error, VA = Department of Veterans Affairs.

*Address all correspondence to Ronald A. Schuchard, PhD; Atlanta RR\&D Center, 151R, 1670 Clairmont Rd, Decatur, GA 30033; 404-728-5063; fax: 404-728-4837.

Email: rschuch@emory.edu

DOI: 10.1682/JRRD.2007.08.0132 
Some who have fitted reading performance data have performed a logarithmic transformation of the reading rate measure before making a spline fit [6]. This transformation introduces nonlinearities into data that are typically viewed as normally distributed about their population means. We, instead, chose to regress data onto functions of monotonic growth. Three monotonic growth models of sigmoidal function were examined: Weibull, Logistic, and Gompertz. Each model has three parameters that specify asymptotic maximum $\{a\}$, shape $\{b\}$, and position $\{c\}$. These models were chosen because their ordinate values at $X=\{c\}$ $\left(Y\left[X_{c}\right]\right)$ were complementary about the half-maximum: $Y\left[X_{c}\right]=0.63 \times\{a\}$ for the Weibull, $Y\left[X_{c}\right]=0.50 \times\{a\}$ for the Logistic, and $Y\left[X_{C}\right]=0.37 \times\{a\}$ for the Gompertz. (Curly braces " \{\} ” specify characteristics and parameters of reading performance.) Each model simulates one of three types of reading rate growth: (1) early, slow acceleration with later, rapid deceleration to a large print size that maintains maximum reading rate plateau (the Weibull); (2) early acceleration symmetric with later deceleration (the Logistic); and (3) early, rapid acceleration with later, slow deceleration (the Gompertz). In analysis 1 , we compared the three models using actual reading performance data from older adults.

In many readers, however, maximum reading rate often occurs at a print size less than $1.30 \log$ MAR (largest print size for Minnesota Low Vision Reading Acuity [MNREAD] chart). Reading rate, therefore, would plateau to a maximum over a range of print sizes and then might decline at the largest print sizes. Modeling reading rate performance could be constructed as the difference of two monotonic functions and be the algebraic sum of increase and decrease in reading rate with print size increase. With this nonmonotonic template, we could calculate characteristics like reading rate maximum, range of print size over which the reader manifests reading rate that is irresolvable from maximum (plateau), and reading rate slopes at print sizes that delimit the plateau.

One-hundred-thirty-two older adults age 65 to 91 years (mean $=75.6)$ completed the MNREAD test [1-2] at the Atlanta Department of Veterans Affairs (VA) Rehabilitation Research and Development Center of Excellence. All participants completed informed consent and Health Insurance Portability and Accountability Act authorization per Atlanta VA and Emory University human subjects requirements. Participants were screened for dementia using the MiniMental State Examination; all participants self-reported normal ability to read and reported having received 3 to
18 years of formal education (mean $=10.36$ ). Visual acuity among them ranged from -0.15 to $0.64 \log$ MAR (median $=$ 0.15) as tested with the Early Treatment Diabetic Retinopathy Study chart. Contrast sensitivity using the Peli-Robson Contrast Sensitivity Chart ranged from 1.05 to 2.05 logcontrast $($ median $=1.74)$. Humphrey visual field testing confirmed normal central visual fields. Therefore, all our subjects had normal age-related visual function. Onehundred-twenty-one subjects returned a second time more than 1 month later to repeat the MNREAD test.

The MNREAD test comprises sentences of 60 characters (average length of 10 words) presented at $40 \mathrm{~cm}$ distance in print size decrements of about 26 percent (-0.10 logMAR) starting at Snellen size 20/400 (1.30 $\log$ MAR) [1-2]. Reading time was measured with a stopwatch and misread words (errors) were recorded. If 10 or more errors occurred, reading rate was recorded as 0 . Sentences of progressively smaller character size proceeded until a subject took longer than 20 seconds (reading rate under $30 \mathrm{wpm}$ without error) to complete a sentence.

Reading time and reading error were transformed into reading rate (units of wpm) and size of textual material and reading distance were transformed into print size (units of logMAR) [1-2]. The 132 older adults created 253 data sets, each a sequence of reading rate-print size pairs that ranged from the smallest readable print size to 1.30 logMAR (“conventional data set length”).

\section{ANALYSIS 1: MONOTONIC REGRESSION OF CONVENTIONAL LENGTH DATA SET}

\section{Methods}

Using commercial software $\left(\mathrm{JMP}^{\circledR}{ }^{\circledR}\right.$, version 5 , SAS Institute; Cary, North Carolina), we regressed each reader's data with Weibull, Logistic, and Gompertz models [5]. Regression was defined as successful if convergence onto a given data set occurred within no more than 150 iterations of the JMP nonlinear modeling platform. Each model had three parameters, determining asymptotic maximum $\{a\}$, shape $\{b\}$, and location $\{c\}$. The three monotonic models Appendix Equations 1(a)-(c) and their initial values Appendix Equations 4-6(c) are given in the Appendix (available online only at www.rehab.research.va.gov/jour/ 08/45/6/pdf/contents.pdf).

The models are all sigmoidal, with bell-shaped derivatives Appendix Equations 2(a)-(d) describing change in reading rate with increasing print size $(X)$. Evaluated at 
inflexion $\{i\}$ for Weibull (Appendix Equations 2(a)(b)), Logistic (Appendix Equation 2(c)), and Gompertz (Appendix Equation 2(d)) models, the derivative gives tangent slope (reading rate/print size) in units of wpm/ logMAR. (Inflexion is where reading rate has zero acceleration with increasing print size; for reading performance, it is where growth in reading rate changes from positively accelerating to negatively accelerating.) For the Logistic and Gompertz (but not the Weibull) models, tangent slope is the same at both $\{i\}$ and $\{c\}$.

The three models are fundamentally different at $\{i\}$. Inflexion ordinate value $\left(Y_{i}\right)$ is $0.50 \times\{a\}$ for the Logistic model, $0.37 \times\{a\}$ for the Gompertz model, and 0.62 for the Weibull model. (For the Weibull, $Y_{i} \rightarrow 0.63 \times\{a\}$ as $\{b\} \rightarrow \infty)$. In other words, as the independent variable (e.g., print size) increases, Gompertz inflexes first, then Logistic, then Weibull. Near $Y=0$ (e.g., at small print size), acceleration is greatest for Gompertz, less for Logistic, and least for Weibull. However, near $Y=\{a\}$ (e.g., at large print size), this order is reversed.

The assumption of univariant data normality was tested on grouped residuals and received limited support. (Grouped residuals are the mean differences between individual reading rate and Weibull regression at each print size averaged over all regressed data sets.) Only at the nine largest print sizes (0.50-1.30 logMAR) was residual normality supported at $p=0.05$ (Wilk-Shapiro). Analysis of variance showed that no significant $(p[F]=0.05)$ change was found in residual variance with regression mean only for the eight largest print sizes (0.60-1.30 logMAR. Assuming correspondence between group and individual means (the group is a cohort of normal individuals), these results suggest that grouped regression values at the eight largest print sizes simulate means of individual univariant and normal distributions. At smaller print sizes, however, this assumption is unsupported. Therefore, we conclude that single reader uncertainty cannot be estimated from our grouped reader response.

Error in the experimenter's response, however, can be used to estimate the reader's uncertainty. Noise variation in simple reaction time response is reported to be constant [7]. When key press is used, subjects respond with a constant uncertainty of $50 \mathrm{~ms}$ at each of a range of stimulus intensity levels. In our reading performance study, reading time was obtained with a stopwatch that required two key presses per measure, at the start and stop of reading. The largest positive recording time error would result from maximum experimenter anticipation (key press $50 \mathrm{~ms}$ before reading onset, which adds $+50 \mathrm{~ms}$ to the response) followed by maximum experimenter delay (key press $50 \mathrm{~ms}$ after reading completion, which again adds $+50 \mathrm{~ms}$ to the response), for a total error of $+100 \mathrm{~ms}$. The largest negative recording time error would result from maximum experimenter delay (key press $50 \mathrm{~ms}$ after reading onset, which adds $-50 \mathrm{~ms}$ ) followed by maximum experimenter anticipation (key press $50 \mathrm{~ms}$ after reading completion, which again adds $-50 \mathrm{~ms}$ ), for a total error of $-100 \mathrm{~ms}$.

To specify experimenter uncertainty, we added two constants $( \pm 100 \mathrm{~ms})$ to each individual reading time value, transformed the data into rate units, and performed the regression. If regression is a psychometric template that simulates reading rate distribution means at each print size, then it is invariant with reader, but not experimenter, uncertainty. Our protocol gave numerical estimates of lower and upper uncertainty bounds of individual maximum reading rate $\left(-\left\{a_{\text {unc }}\right\}\right.$ and $\left.+\left\{a_{\text {unc }}\right\}\right)$. Intersection of $-\left\{a_{\text {unc }}\right\}$ with the regression function gave $\{\mathrm{CPS}\}$. Figure 1(a) shows this response in a typical reader.

Regression by Appendix Equation 1(a) produced the monotonic reading performance template, with asymptotic maximum $\{a\}$, slope $\left\{d Y / d X_{i}\right\}$, and $\{C P S\}$ (abscissa projection of intersection by $-\left\{a_{\text {unc }}\right\}$ and Appendix Equation 1(a)).

\section{Results}

We wanted to choose the best model to serve as a template for monotonic reading performance. Our criterion was greatest frequency of convergence at lowest residual error both within (squared residuals [SR]) and across (sum squared error [SSE]) print size. Then, averaged over all regressed data sets-

1. Weibull regression had the highest frequency of data set convergence at 248/253 (98\%), followed by Logistic (92\%), and then Gompertz (91\%).

2. Model difference in SSE was $<1$ percent.

3. For print sizes between 0.40 and $1.30 \log$ MAR, model difference in SR was $<0.2$ percent. Figure 1(b) shows that 80 percent of $\{\mathrm{CPS}\}$ (obtained using the Weibull) was found at or above $0.30 \log M A R$. At 0.30 logMAR, Weibull regression showed greater SR (Figure 2 (b)).

These findings justified use of the Weibull model.

Five characteristics of reading performance regression were obtained (shown in Figure 1(a)): $\{a\},\left\{a_{\mathrm{unc}}\right\}$, $\left\{d Y / d X_{i}\right\},\{C P S\}$, and abscissa at inflexion $\left(X_{i}\right)$. The first 


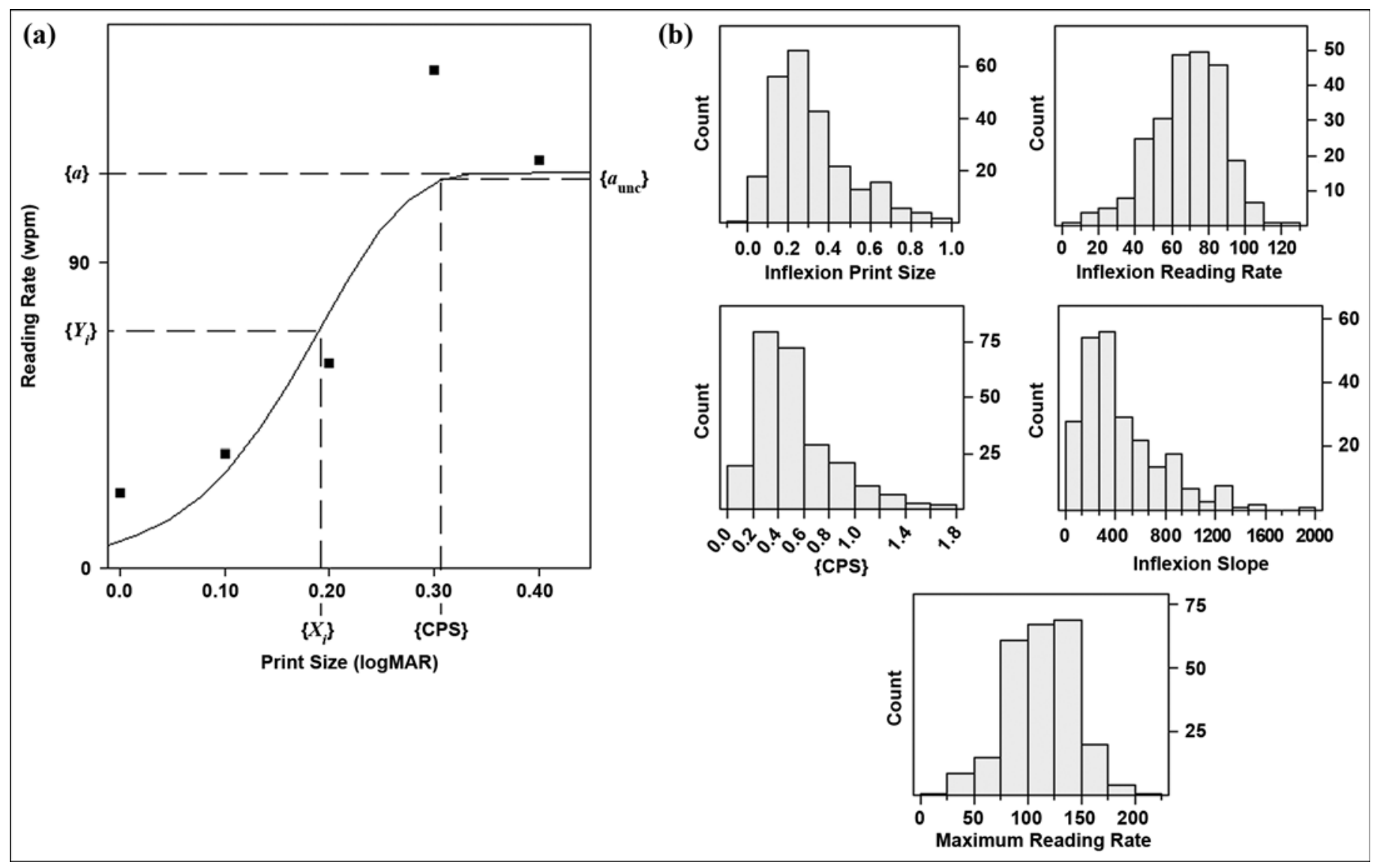

Figure 1.

(a) Detail of Weibull regression (Appendix Equation 1(a), see main text). Shown are reading rate inflection coordinates $\left(\left\{X_{i}, Y_{i}\right\}=0.195\right.$ logMAR, 89.9 wpm), uncertainty at maximum reading rate $\left(\left\{a_{\mathrm{unc}}\right\}=114.1 \mathrm{wpm}\right)$, critical print size $\left.(\{\mathrm{CPS}\}=0.31 \operatorname{logMAR}\}\right)$, and maximum reading rate $(\{a\}=116.4$ wpm). Note that slope at inflexion $\left(\left\{d Y / d X_{i}\right\}=553 \mathrm{wpm} / \operatorname{logMAR}\right)$ has changed from its initial value $(Y / X=115 \mathrm{wpm} / \log \mathrm{gAR})$. (b) Distributions of monotonic characteristics across 247 data sets. Units of inflexion print size and $\{\mathrm{CPS}\}$ are in logMAR; units of inflexion and maximum reading rates are in wpm; and units of inflexion slope are in wpm/logMAR.

four have already been defined: $X_{i}$ was determined by setting the second derivative of Appendix Equation 1(a) equal to zero and solving for $X$; substitution into Appendix Equation 1(a) gave $Y_{i}$.

For the data used in Figure 2, characteristics are $\left\{X_{i}\right.$, $\left.Y_{i}\right\}=0.195 \operatorname{logMAR}, 89.9 \mathrm{wpm},\left\{d Y / d X_{i}\right\}=553 \mathrm{wpm} /$ $\operatorname{logMAR},\{\mathrm{CPS}\}=0.306 \operatorname{logMAR}$, and $\{a\}=116 \mathrm{wpm}$. Only 247 of 253 data sets could be regressed because 6 sets did not possess sigmoidal growth characteristics that would permit successful regression onto Appendix Equation 1(a). Five of these sets never reached a plateau (average maximum reading rate), and one set failed to show $\{$ CPS $\}$.

For the others, variation in characteristic values is shown in the five distributions of Figure 1(b). Note the skew. Median inflexion reading rate appears to inversely correspond with its change (inflexion slope) and with \{CPS $\}$. High reading rates across our group of readers with normal age-related vision appear to be associated with a low rate of change and with print size values near rate maximum.

\section{ANALYSIS 2: MONOTONIC REGRESSION OF SHORTENED LENGTH DATA SET}

\section{Methods}

To improve efficiency of data collection, we examined the effect of shortening data set length. In our shortening protocol, data set length was truncated by reducing 


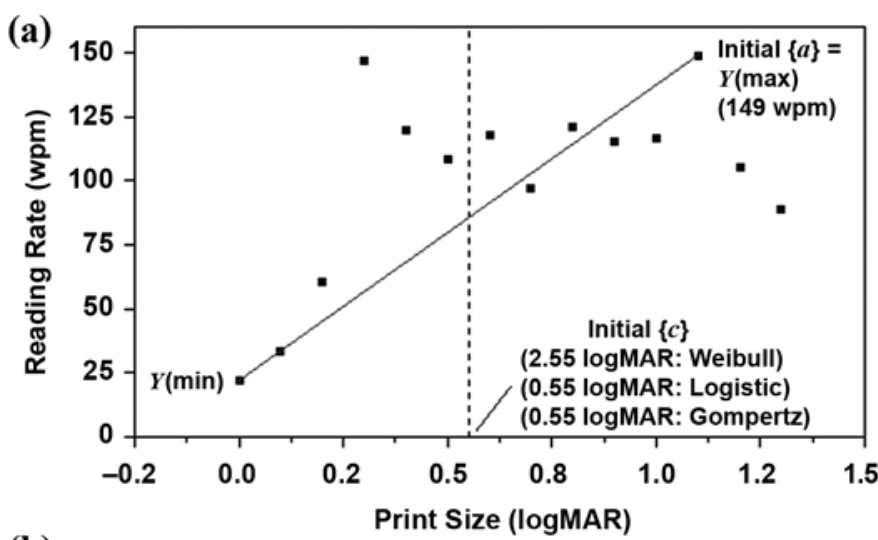

(b)

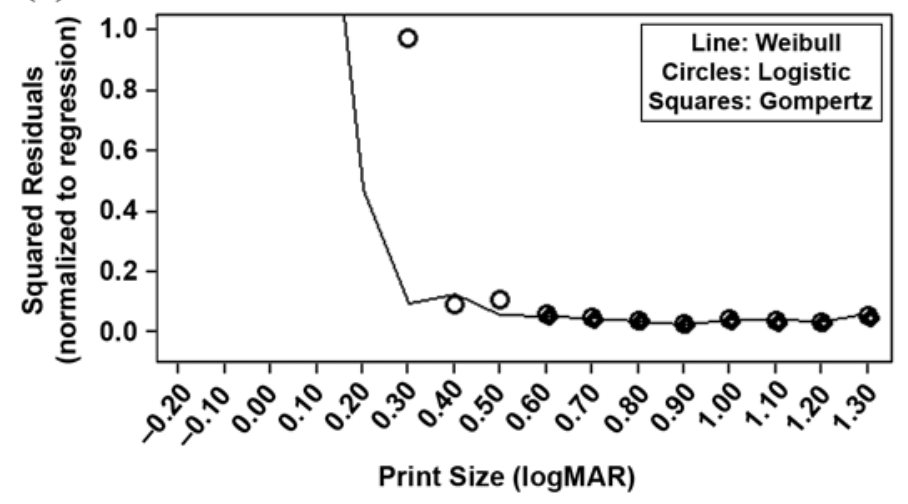

Figure 2.

(a) Procedure for setting initial parameter values for monotonic regression. Line segment between $Y_{\max }$ and $Y_{\min }$ shows slope of change $(Y / X)$. (b) Between 0.30 and $1.30 \log M A R$, regression error using Weibull (line plot) is equal to or less than that obtained using either Logistic (circles) or Gompertz (squares). (Below 0.30 logMAR for Logistic and $0.60 \operatorname{logMAR}$ for Gompertz squared residuals are off-scale.)

the value of the largest print size presented in 0.10 logMAR increments. Data set length was longest when the largest print size was 1.30 logMAR (first tuncation step, which gave the "conventional data set length") and shortest when the largest print size was $0.70 \log$ MAR (seventh truncation step, which gave the "shortest data set length").

All data sets used in the previous section (247) were considered. Successive truncation was performed only if two conditions were met: (1) response was present at every data set length and (2) at least five reading rate responses comprised the shortest data set length. These conditions were satisfied by 204 data sets. Truncation of each gave 7 data subsets with maximum print sizes ranging from 1.30 to $0.70 \operatorname{logMAR}$, for a total of $1,428(204 \times 7)$ data subsets. Each subset was then individually regressed onto Appen- dix Equation 1(a) and analyzed, both by group (all subsets averaged within truncation step) and by individual subset.

Grouped parameter error ranges at each truncation step were then computed by considering the effect of noise variation in simple reaction time response [7]. This entailed (1) summing one of two constants ( $\pm 100 \mathrm{~ms}$ ) onto reading time values in each conventional length data set (see methodolgy of analysis 1 for justification), (2) converting units to reading rate, (3) regressing each set, and (4) averaging normalized parameter values. This protocol gave three error ranges: $\pm\{a\}, \pm\{b\}$, and $\pm\{c\}$. If a normalized parameter value from a shortened length data set fell within the conventional length data set error range, we assumed it was noise deviation.

Grouped residual error (root-mean-square normalized error [RMSNE]) was also computed at each truncation step by averaging normalized residual errors of regression across data set and print size above $\{\mathrm{CPS}\}$. Because it is a group response, RMSNE can only indicate change in variance (along the reading rate asymptotic plateau). It would only suggest (at least for some readers) the possibility of departure from sigmoidal behavior at large print size.

\section{Results}

The effect on grouped parameter values of shortening data set length is shown in Figure 3. Group error limits for $\{a\}$ (horizontal lines) are similar for $\{b\}$ and are about one-twentieth as large for $\{c\}$.

While the group value of $\{a\}$ remains within these error limits as data set length is shortened, those of $\{b\}$ and $\{c\}$ do not. At the shortest data set length, the group value of $\{b\}$ increases by 70 percent of its average value at conventional length while the group value of $\{c\}$ decreases 99 percent. Therefore, shortening data set length results in significant parameter change. Furthermore, goodness of linear fit $\left(R^{2}\right)$ for individual data set values of $\{a\}$ between conventional and shortest data set length is poor $\left(R^{2}=0.61\right)$. This finding suggests that individual data set variation in $\{a\}$ might be averaged out when calculating group means.

Parameters $\{b\}$ and $\{c\}$ and characteristics $\{d Y /$ $\left.d X_{i}\right\}$ and $\{\mathrm{CPS}\}$ are compared in Figure 4.

Here, slope (closed squares) and intercept (open circles) of linear fit between $\{b\}$ and $\left\{d Y / d X_{i}\right\}$ (Figure 4(a)) or $\{c\}$ and $\{\mathrm{CPS}\}$ (Figure 4(b)) are roughly constant as data set length is shortened. In Figure 4(c), $R^{2}$ for $\{b\}$ versus $\left\{d Y / d X_{i}\right\}$ varies between 0.90 and 0.94 (left axis), while $R^{2}$ for $\{c\}$ versus $\{$ CPS $\}$ varies between 0.78 and 0.83 


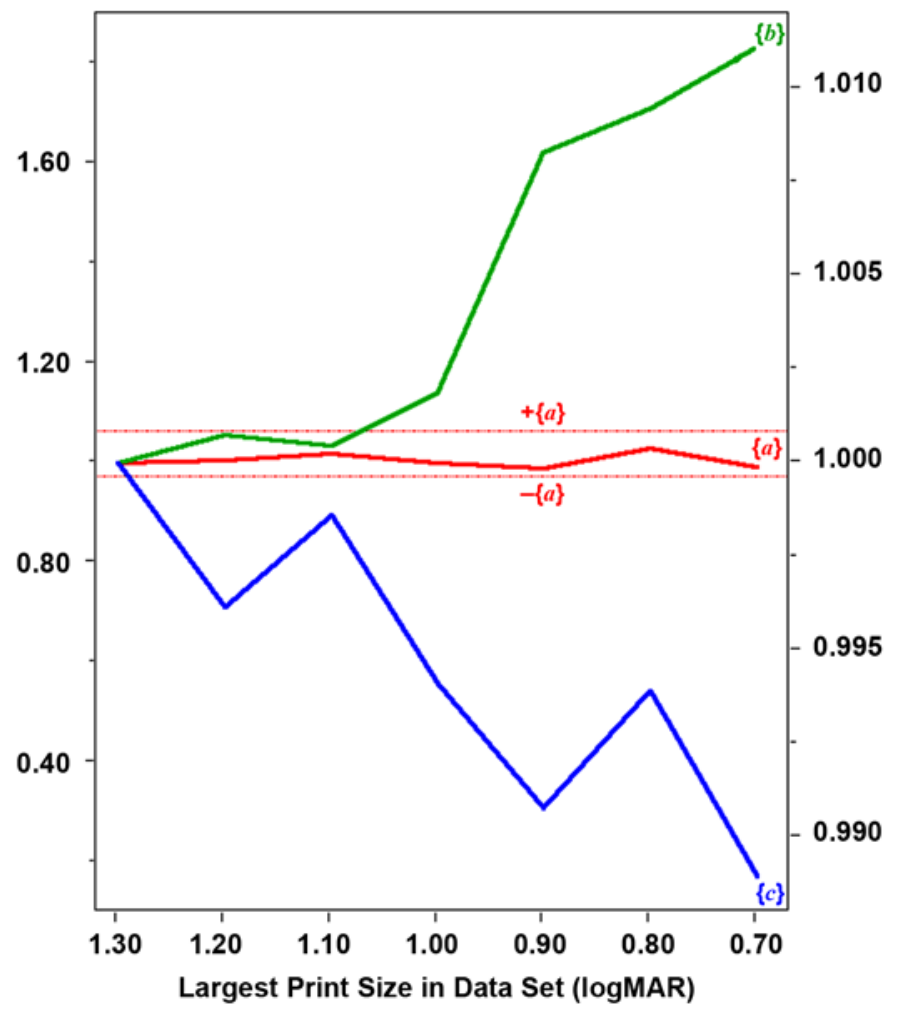

Figure 3.

Change in average normalized parameter value with decreasing data set length. Left ordinate is for $\{a\}$ and $\{b\}$; right ordinate is for $\{c\}$. Horizontal lines about $\{a\}(+\{a\},-\{a\})$ are explained in main text.

(right axis), suggesting predictive validity (across data set length) only for $\{b\}$ and $\left\{d Y / d X_{i}\right\}$.

Figure 5 shows that RMSNE decreases with decrease in data set length (largest print size) with significant correlation $(r=0.945, p=0.01)$ [8].

Figure 5 indicates increased variation in reading rate at large print size. In group response, this increase suggests the presence of subgroups that might respond differently at those sizes. One subgroup might gradually approach (negatively accelerate to) maximum rate while a second subgroup approaches more rapidly (positively accelerates) and a third declines. We next test this possibility.

\section{ANALYSIS 3: NONMONOTONIC REGRESSION OF CONVENTIONAL LENGTH DATA SET}

\section{Methods}

To examine reading rate variation at large print size, we modified Appendix Equation 1(a) to permit nonmonoto- (a)

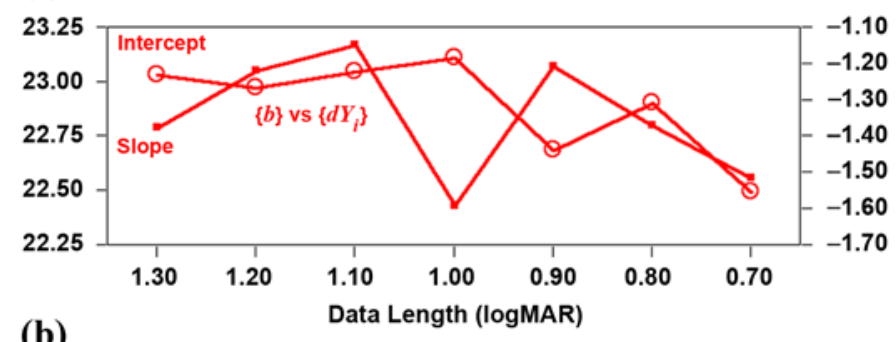

(b)

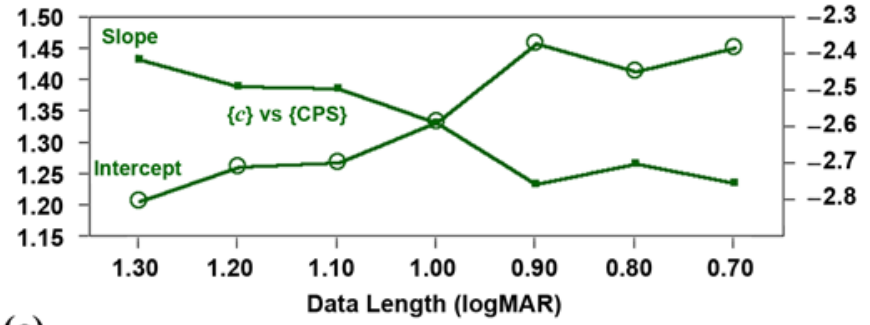

(c)

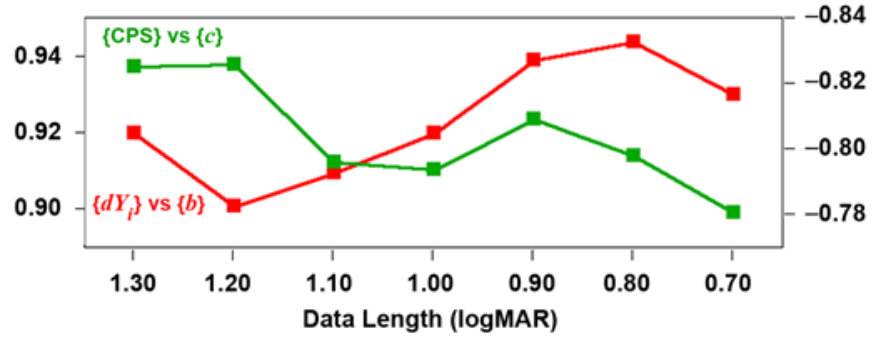

Figure 4.

Intercept (open circles, right-hand ordinates in (a)-(b)) and slope (closed squares, left-hand ordinates in (a)-(b)) of linear fit taken across data length for (a) $\{b\}$ versus $\left\{d Y / d X_{i}\right\}$ and (b) $\{c\}$ vs $\{C P S\}$. (c) Shows $R^{2}$ for $\{b\}$ versus $\left\{d Y / d X_{i}\right\}$ (left ordinate) and for $\{c\}$ versus $\{\mathrm{CPS}\}$ (right ordinate). $\mathrm{CPS}=$ critical print size.

nicity. Mixed-additive (nonmonotonic) Weibull models (Appendix Equations 9(a) and (c)) were used [9], giving nonmonotonic templates of reading performance that had five parameters $\{a, b, c, g, e\}$.

Appendix Equation 9(a) is the sigmoidal sum and Appendix Equation 9(b) the sigmoidal difference of two monotonic Weibull models (Appendix Equation 1(a)). Rearrangement gives Appendix Equation 9(c). The first term represents increase in reading rate with increase in print size. The second term represents either further increase $(+)$ or decrease $(-)$ in rate. Appendix Equations 1(a) and 9(a-b) specify reading rate that asymptotically approaches maximum (Equation 1(a)), nonasymptotically approaches maximum (Appendix Equation 9(a)), or declines from maximum (Appendix Equation 9(b)). 
Initial parameter values for the nonmonotonic models are given in the Appendix. Regression was defined as successful if convergence occurred within no more than 150 iterations of the JMP nonlinear modeling platform.

\section{Results}

Nonmonotonicity (regressibility by Appendix Equation 9(b)) was shown by 111 of 248 conventional length sigmoidally regressible data sets $(111 / 248=45 \%)$. Of these 111, 49 percent had lower mean SRs (SRs divided by appropriate degrees of freedom) than when either asymptotic (Appendix Equation 1(a)) or monotonically increasing (Appendix Equation 9(a)) models were used. This finding indicates that 22 percent $(45 \% \times 49 \%)$ of our 248 sigmoidally regressible data sets gave evidence of reduction in reading rate at large print size (falloff). The remainder $(100 \%-22 \%=78 \%)$ had apparently not yet reached maximum reading rate at the largest print size.

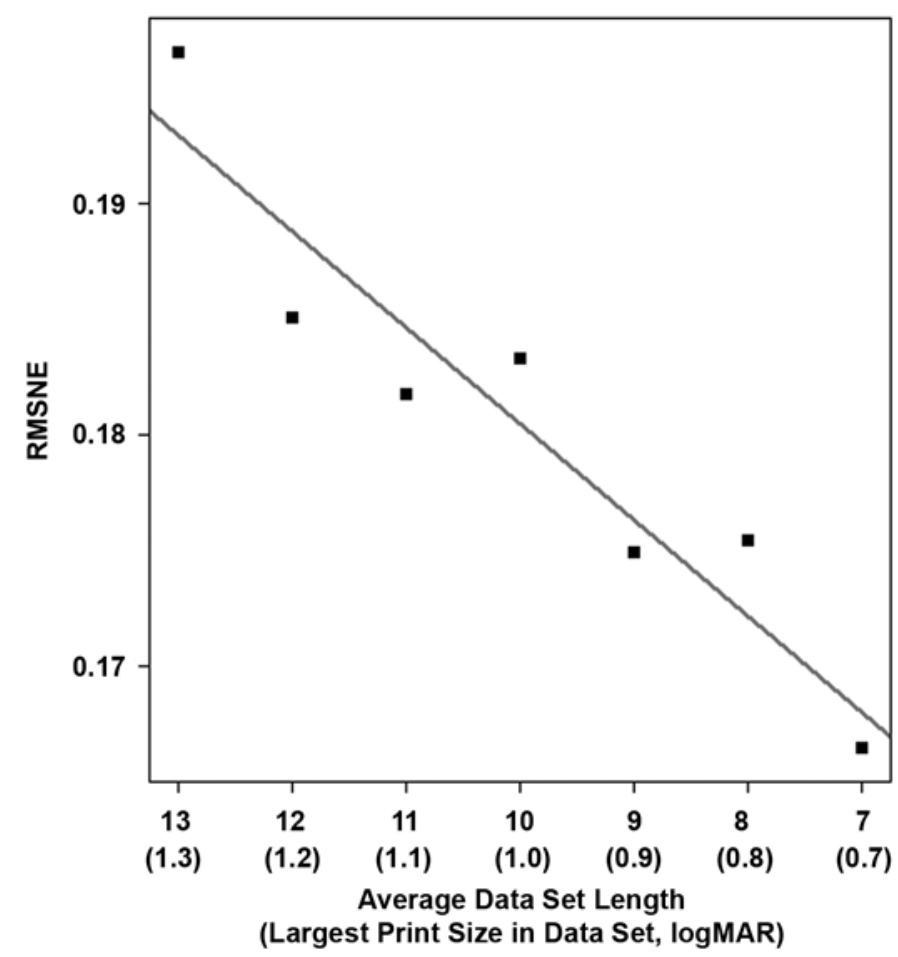

Figure 5.

Normalized regression error (root-mean-square normalized error [RMSNE]) for print sizes above \{CPS \} averaged across 204 data sets versus data set length. Also shown is largest print size corresponding to each data set length (in parentheses) and line of best fit between RMSNE and data set length (largest print size). CPS = critical print size.
The falloff subgroup provided eight characteristics: (1) average maximum reading rate ( $\{\mathrm{Max}\}$, determined by numerical interpolation of regression); (2) uncertainty at maximum $\left(\left\{\operatorname{Max}_{\text {unc }}\right\}\right.$, determined by multiplying $\{\operatorname{Max}\}$ into $\left.\left\{a_{\text {unc }}\right\} /\{a\}\right)$; (3)-(4) abscissa projections at their intersection ( $\left\{X_{1}\right\}$ and $\left.\left\{X_{2}\right\}\right)$; (5) print size range $\left(\left\{X_{2}\right\}-\left\{X_{1}\right\}=\right.$ \{plateau $\}$ ), over which reading rate was effectively maximum (between $\left\{\operatorname{Max}_{\mathrm{unc}}\right\}$ and $\{\mathrm{Max}\}$ ); (6)-(7) derivatives of Appendix Equation 9(a) at abscissa limits $\left(\left\{d Y / d X_{1}\right\}\right.$ and $\left.\left\{d Y / d X_{2}\right\}\right)$; and (8) reading rate at $1.30 \log M A R$, or falloff ( $\{\mathrm{FO}\})$ (determined by substitution).

Figure 6 shows these characteristics for the typical subject, with the following values: $\left\{X_{1}\right\}=0.31 \log$ MAR; $\left\{d Y / d X_{1}\right\}=112.1 \mathrm{wpm} / \operatorname{logMAR} ;\{\operatorname{Max}\}=120.0 \mathrm{wpm}$; \{plateau $\}=0.84 \operatorname{logMAR} ;\left\{\operatorname{Max}_{\text {unc }}\right\}=117.6 \mathrm{wpm} ;\{d Y /$ $\left.d X_{2}\right\}=-43.2$ wpm/logMAR; $\left\{X_{2}\right\}=1.15 \operatorname{logmar} ;\{$ FO $\}=$ $87.8 \mathrm{wpm}$; and $\{\mathrm{FO}\} /\{\mathrm{Max}\}=0.73$.

Data sets that successfully regressed onto Appendix Equation 9(b) gave distributions for these characteristics. Figure 7 shows distributions for $\left\{X_{1}\right\},\left\{d Y / d X_{1}\right\}$, $\{\operatorname{Max}\}$, \{plateau $\},\left\{\operatorname{Max}_{\mathrm{unc}}\right\},\left\{d Y / d X_{2}\right\},\left\{X_{2}\right\}$, and $\{\mathrm{FO}\} /$ $\{$ Max $\}$ at $1.30 \log M A R$.

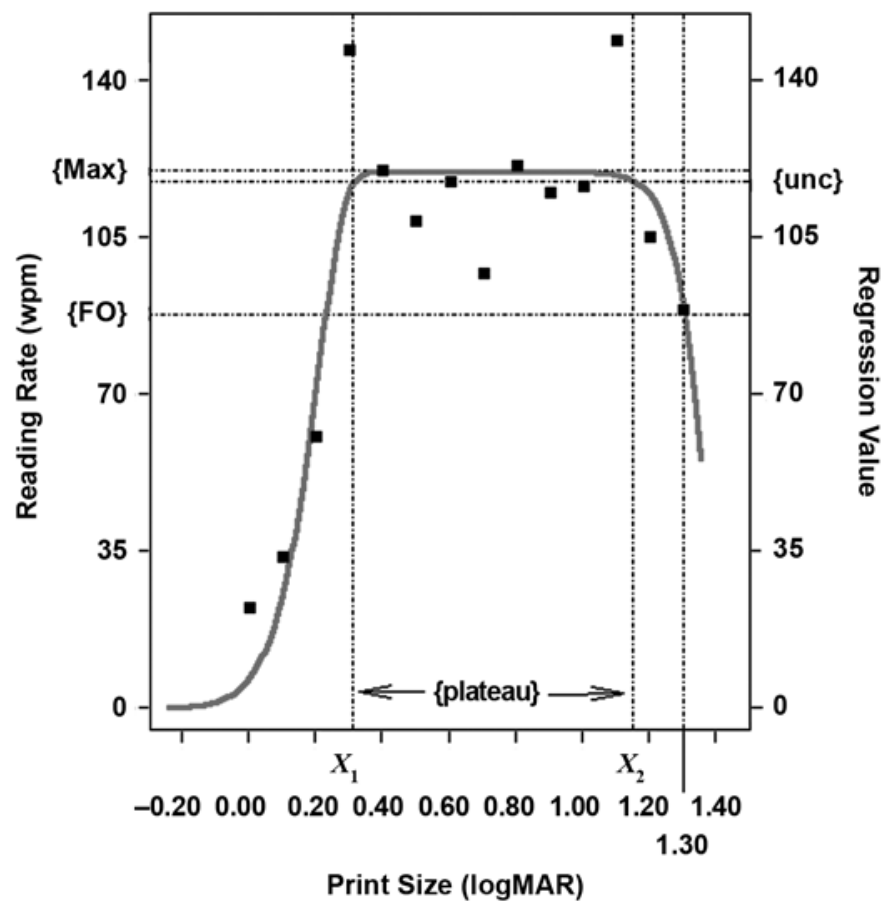

Figure 6.

Location of nonmonotonic Weibull characteristics on data of typical reader: maximum $(\{\operatorname{Max}\})$, uncertainty of maximum ( $\{\mathrm{unc}\}$ ), corresponding abscissa projections $\left(\left\{X_{1}\right\}\right.$ and $\left.\left\{X_{2}\right\}\right)$ for points of intersection with Appendix Equation 9(a) (see main text), \{plateau\}, and falloff (\{FO $\})$ at $1.30 \log M A R$. 
(a)

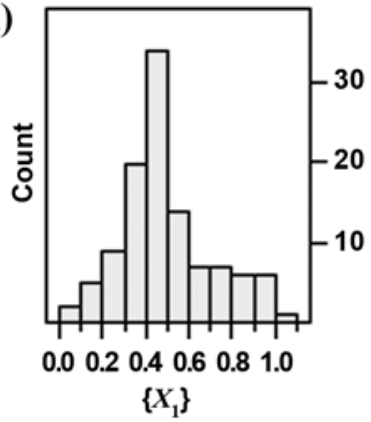

(d)

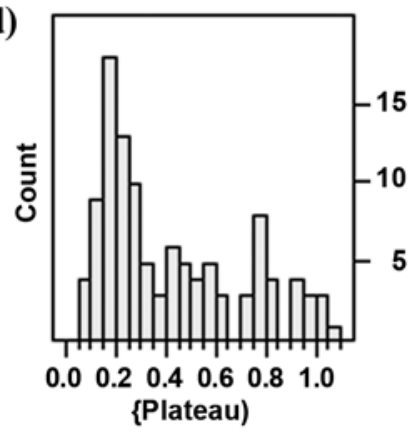

(g)

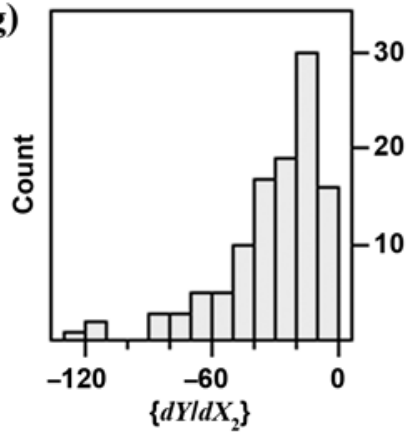

(b)

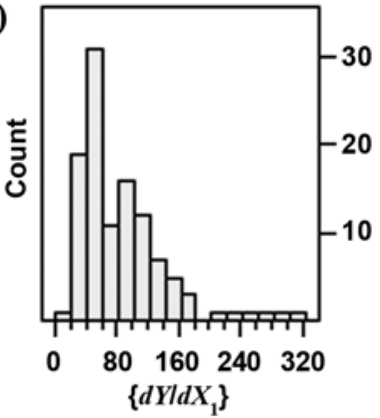

(e)

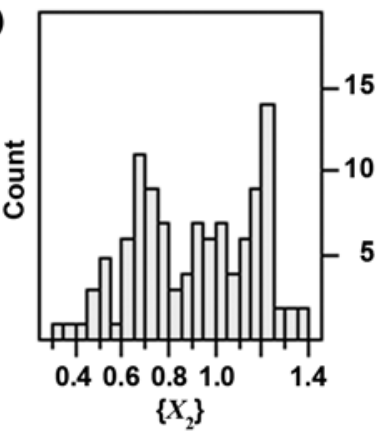

$\left\{X_{2}\right\}$ (c)

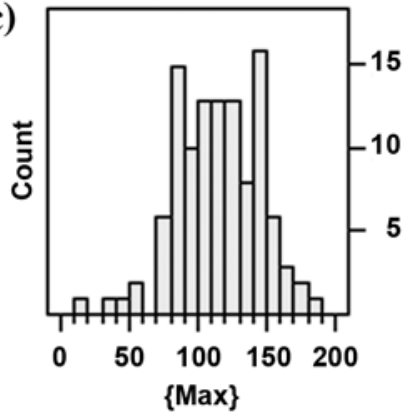

(f)

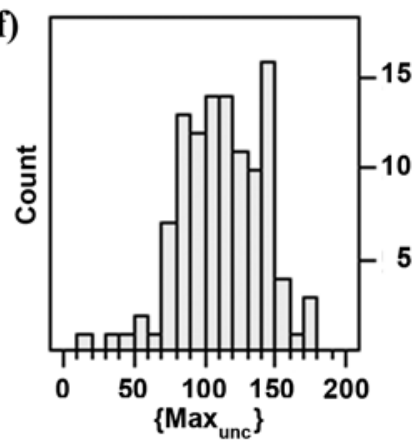

(h)

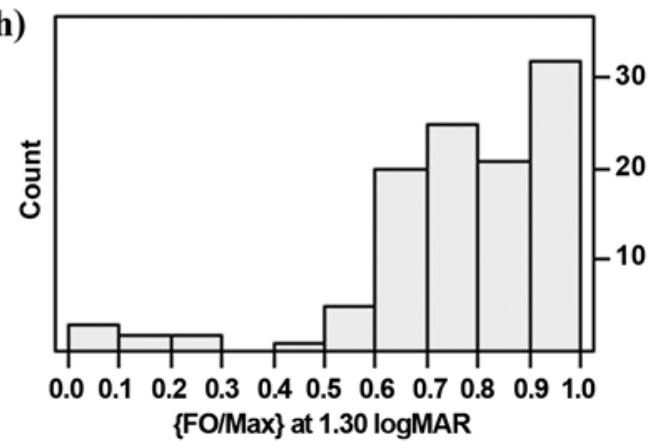

Figure 7.

Distributions of nonmonotonic characteristics across 111 data sets: (a) $\left\{X_{1}\right\}$, (b) $\left\{d Y / d X_{1}\right\}$, (c) $\{\operatorname{Max}\}$, (d) $\{$ plateau $\}$, (e) $\left\{X_{2}\right\}$, (f) $\{$ Max $\mathrm{unc}\}$, (g) $\{d Y /$ $\left.d X_{2}\right\}$, and (h) estimates of $\{\mathrm{FO} / \mathrm{Max}\}$ at $1.30 \log \mathrm{MAR}$. Units of $\left\{X_{1}\right\}$, \{plateau $\}$, and $\left\{X_{2}\right\}$ are in logMAR; units of $\{$ Max $\}$ and $\{$ Max $\mathrm{unc}\}$ are in wpm; units of $\left\{d Y / d X_{1}\right\}$ and $\left\{d Y / d X_{2}\right\}$ are in wpm/logMAR; $\{\mathrm{FO} / \mathrm{Max}\}$ is unitless. FO = falloff, Max = maximum, Max unc $=$ uncertainty of maximum.

The following four observations can be made:

1. Absolute values of $\left\{d Y / d X_{1}\right\}$ appear to cluster around $50 \mathrm{wpm} / \log \mathrm{MAR}$, while those for $\left\{d Y / d X_{2}\right\}$ cluster about $20 \mathrm{wpm} / \log M A R$. Their ratio $\left(\left|\left\{d Y / d X_{1}\right\}\right| /\{\mid d Y /\right.$ $\left.d X_{2}\right\} \mid=2.5$ ) indicates that the preplateau rising slope $\left(\left\{d Y / d X_{1}\right\}\right)$ greatly exceeds the postplateau falling slope $\left(\left\{d Y / d X_{2}\right\}\right)$. Thus, maximum reading rate in normal subjects appears to be attained more quickly at smaller print size, while decrease in reading rate at large print size is relatively muted.

2. $\{$ Plateau $\}$ correlates more with $\left\{X_{2}\right\}$ than with $\left\{X_{1}\right\}$, suggesting that increase in $\left\{X_{2}\right\}$ (not decrease in $\left\{X_{1}\right\}$ ) is responsible for increase in \{plateau $\}$.
3. Note the similarity between monotonic and rising portion of nonmonotonic Weibull regressions (Figures 2(b) and 7, respectively). Median values of $\left\{d Y / d X_{i}\right\}$ (343.0 wpm/ $\log$ MAR), \{CPS $\}$ (0.435 $\operatorname{logMAR}$ ), and $\{a\}$ (112.0 wpm) given by Appendix Equation 1(a) approximate (respectively) those of slope at print size of monotonic inflexion (340.3 wpm/logMAR), $\left\{X_{1}\right\}$ (0.440 logMAR), and \{Max (115.5 wpm) given by Appendix Equation 9(b). This is to be expected because the first term of Appendix Equation 9(b), predominant at small print size, is Appendix Equation 1(a).

4. The last distribution (Figure 7(h)) establishes a numerical criterion for reading rate falloff at $1.30 \log$ MAR 
( $\{\mathrm{FO}\}$ ). Let us define falloff criterion (normalized to $\{\operatorname{Max}\})$ as $\{\mathrm{FO}\} /\{\mathrm{Max}\}$ at the 5 percent quantile (where the value of $\{\mathrm{FO}\} /\{\mathrm{Max}\}$ is smaller than that given by 95\% of all data sets). Furthermore, if Appendix Equation 9(b) fails to regress, let $\{\mathrm{FO}\} /\{\operatorname{Max}\}=1$. Then, $\{\mathrm{FO}\} /\{\mathrm{Max}\}=57$ percent, i.e., $\{\mathrm{FO}\}=57$ percent of $\{\mathrm{Max}\}$. If a reader of unknown provenance gives a lower value, we shall conclude that his reading rate is significantly under criterion for a member of a group of subjects with normal vision and should be flagged as clinically problematic.

\section{DISCUSSION}

Spline fits of reading rate on logarithmically scaled ordinates have been reported [7]. This procedure, however, complicates determination of average maximum reading rate and $\{\mathrm{CPS}\}$ by its direct data reference and nonlinearity of ordinate scaling; the latter distorts the hypothetical data distribution. Weibull regression on linearly scaled ordinates has already been demonstrated [10], as have results comparing subjects with normal vision against patients in a very small group of older adults with normal age-related vision [11]. Our study shows that the Weibull regression model can fit all the appropriate reading performance data from older adults.

Regression by a nonmonotonic Weibull model indicates that reading rate might sometimes decline at large MNREAD chart print sizes designed for older adults with normal age-related vision. This concept is not new as Legge et al. showed that reading rates for young adults (in their twenties) gradually decreased at character sizes larger than $2^{\circ}$ [12]. However, the MNREAD charts were designed so normally sighted readers were expected to maintain average maximum reading rate even at the large character sizes (the largest lowercase letter on a MNREAD chart subtends a visual angle of about $1.7^{\circ}$ at the standard viewing distance, $40 \mathrm{~cm}$ ). While our results indicate that reading rates at large print sizes may decline even for normally sighted older adults, other reading studies have assumed this is a low vision characteristic [12].

The combination of visual, lexical, and oculomotor information has been integrated into an ideal-reader model of reading [13]. Here, the concept of the visual span (the number of characters that can be identified in a single fixation) plays a unifying role. Increased print size decreases this span [14] and suggests a decrease in read- ing rate. Further work, perhaps facilitated by a regression protocol similar to ours, is needed to investigate the relationship between possible declining visual span in older adults and declining reading rates.

Unfortunately, no set standard exists for the reading rate that corresponds to the estimate of $\{\mathrm{CPS}\}$. Previously published studies have equated this value with reading rates ranging from 75 to 99 percent of the average maximum. Besides the measure of experimenter uncertainty that we used to determine $\{\mathrm{CPS}\}$, a number of reader-originated factors that likely contribute to variability are (1) time required to visualize a letter sequence comprising a word or sentence, interpret its meaning, and vocalize it; (2) saccadic eye movements and fixation pauses; and (3) verbal intelligence, attentional maintenance, and self-assurance. Variability in these visual, lexical, and oculomotor factors derives from changing sensory, cognitive, and muscle factors, different within and between each reader. Unlike the experimenter uncertainty used in this study, these factors are difficult to measure but add considerable temporal uncertainty to recorded values of reading time.

Nevertheless, our work has demonstrated use of (1) Weibull monotonic and nonmonotonic functions in quickly assessing normal reader reading rate characteristics; (2) data sets less than conventional length $(-0.20$ to $1.30 \log$ MAR); and (3) a means to objectively assess falloff in reading rate in readers with unknown clinical provenance.

\section{CONCLUSIONS}

\section{Analysis 1}

A monotonic Weibull model gives equal or lower regression error and increased convergence onto data of conventional length than do either Logistic or Gompertz models. Weibull regression gives estimates of reading rate slope and maximum. Furthermore, consideration of uncertainty in the experimenter's response provides an estimate of the $\{$ CPS $\}$.

\section{Analysis 2}

Shortening the data set length by eliminating the largest print sizes of monotonic Weibull regression gives lower average per datum error variation but significantly changes parameter values. 


\section{Analysis 3}

A nonmonotonic Weibull model showing falloff in reading rate at large print size was found superior in some cases to monotonic and nonmonotonic models showing continued reading rate increase. This model gave estimates of maximum reading rate, its uncertainty, location and length of response flatness (plateau), slopes at plateau limits, and reading rate at $1.30 \log$ MAR. The last parameter shows reading rate reduction at the largest print size and provides a criterion measure for evaluating readers of unknown clinical provenance.

\section{ACKNOWLEDGMENTS}

This material was based on work supported in part by VA, Veterans Health Administration, Office of Research and Development, Rehabilitation Research and Development Service merit review grants C3384U to Drs. Nygaard and Schuchard and E2569R to Drs. Echt and Schuchard.

The views expressed in this article are those of the authors and do not necessarily represent the views of the VA or U.S. Government.

The authors have declared that no competing interests exist.

\section{REFERENCES}

1. Legge GE, Ross JA, Luebker A, LaMay JM. Psychophysics of reading. VIII. The Minnesota Low-Vision Reading Test. Optom Vis Sci. 1989;66(12):843-53. [PMID: 2626251]

2. Mansfield JS, Ahn SJ, Legge GE, Luebker A. A new reading-acuity chart for normal and low vision. OSA Technical Digest. 1993;3:232-35.
3. Laming DR. Mathematical psychology. London (England): Academic Press; 1973. p. 140.

4. Laming DR. Sensory analysis. London (England): Academic Press; 1986. p. 40.

5. Draper NR, Smith H. Applied regression analysis. 3rd ed. New York (NY): Wiley; 1998. p. 544-49.

6. Mansfield JS, Legge GE, Bane MC. Psychophysics of reading. XV: Font effects in normal and low vision. Invest Ophthalmol Vis Sci. 1996;37(8):1492-1501. [PMID: 8675391]

7. Luce RD, Bush RR, Galanter E. Handbook of mathematical psychology 1. Chapters 1-8. New York (NY): Wiley; 1963. p. 319.

8. Sachs L. Applied statistics: A handbook of techniques. New York (NY): Springer-Verlag; 1984. p. 424-25.

9. Murthy DN, Xie M, Jiang R. Weibull models. Hoboken (NJ): Wiley; 2004. p. 11, 28.

10. Kallie CS, Cheung SH, Legge GE, Owsley C, McGwin G. Nonlinear mixed effects modeling as an estimation procedure for sparse MNREAD data. Invest Ophthalmol Vis Sci. 2005;46:E-Abstract 4589.

11. Schuchard RA, Lim J-M, Fletcher DC. Characteristics of reading speed across character size in relation to visual factors. OSA Technical Digest Series. 1998;1:92-95.

12. Legge GE, Pelli DG, Rubin GS, Schleske MM. Psychophysics of reading-I. Normal vision. Vision Res. 1985; 25(2):239-52. [PMID: 4013091]

13. Legge GE, Hooven TA, Klitz TS, Mansfield JS, Tjan BS. Mr. Chips 2002: New insights from an ideal-observer model of reading. Vision Res. 2002;42(18):2219-34. [PMID: 12207981]

14. Legge GE, Cheung SH, Yu D, Chung ST, Lee HW, Owens DP. The case for the visual span as a sensory bottleneck in reading. J Vis. 2007;7(2):1-15. [PMID: 18217824]

Submitted for publication August 27, 2007. Accepted in revised form March 5, 2008. 